\title{
Spin dynamics of edge-sharing spin chains in $\mathrm{SrCa}_{13} \mathrm{Cu}_{24} \mathrm{O}_{41}$
}

\author{
Guochu Deng, ${ }^{1, *}$ Dehong Yu, ${ }^{1}$ Richard Mole, ${ }^{1}$ Ekaterina Pomjakushina, ${ }^{2}$ Kazimierz Conder, ${ }^{2}$ Michel Kenzelmann, ${ }^{3}$ \\ Shin-ichiro Yano, ${ }^{4}$ Chin-Wei Wang, ${ }^{4}$ Kirrily C. Rule, ${ }^{1}$ Jason S. Gardner, ${ }^{4}$ Huiqian Luo, ${ }^{5}$ Shiliang Li, ${ }^{5}$ Clemens Ulrich, ${ }^{6}$ \\ Paolo Imperia, ${ }^{1}$ Wei Ren, ${ }^{7}$ Shixun Cao,${ }^{7}$ and Garry J. McIntyre ${ }^{1, \dagger}$ \\ ${ }^{1}$ Australian Centre for Neutron Scattering, Australian Nuclear Science and Technology Organisation, \\ New Illawarra Road, Lucas Heights, NSW 2234, Australia \\ ${ }^{2}$ Laboratory for Multiscale Materials Experiments, Paul Scherrer Institut, CH-5232 Villigen-PSI, Switzerland \\ ${ }^{3}$ Laboratory for Neutron Scattering and Imaging, Paul Scherrer Institut, CH-5232 Villigen-PSI, Switzerland \\ ${ }^{4}$ National Synchrotron Radiation Research Centre, Hsinchu 30076, Taiwan \\ ${ }^{5}$ Beijing National Laboratory for Condensed Matter Physics, Institute of Physics, Chinese Academy of Sciences, Beijing 100190, China \\ ${ }^{6}$ School of Physics, University of New South Wales, Kensington, NSW 2052, Australia \\ ${ }^{7}$ Department of Physics, International Centre of Quantum and Molecular Structures, and Materials Genome Institute, \\ Shanghai University, Shanghai 200444, China
}

(Received 26 April 2018; revised manuscript received 9 October 2018; published 12 November 2018)

\begin{abstract}
The low-energy magnetic excitation from the highly Ca-doped quasi-one-dimensional magnet $\mathrm{SrCa}_{13} \mathrm{Cu}_{24} \mathrm{O}_{41}$ was studied in the magnetic ordered state by using inelastic neutron scattering. We observed the gapless spinwave excitation, dispersive along the $a$ and $c$ axes but nondispersive along the $b$ axis. Such excitations are attributed to the spin wave from the spin-chain sublattice. Model fitting to the experimental data gives the nearestneighbor interaction $J_{c}$ as $5.4 \mathrm{meV}$ and the interchain interaction $J_{a}=4.4 \mathrm{meV} . J_{c}$ is antiferromagnetic and its value is close to the nearest-neighbor interactions of the similar edge-sharing spin-chain systems such as $\mathrm{CuGeO}_{3}$. Comparing with the hole-doped spin chains in $\mathrm{Sr}_{14} \mathrm{Cu}_{24} \mathrm{O}_{41}$, which shows a spin gap due to spin dimers formed around Zhang-Rice singlets, the chains in $\mathrm{SrCa}_{13} \mathrm{Cu}_{24} \mathrm{O}_{41}$ show a gapless excitation in this paper. We ascribe such a change from gapped to gapless excitations to holes transferring away from the chain sublattice into the ladder sublattice upon Ca doping.
\end{abstract}

DOI: 10.1103/PhysRevB.98.184411

\section{INTRODUCTION}

The dimensionality of a spin system is one of the most important characteristics for its underlying physics. Most of the three-dimensional (3D) Heisenberg magnetic systems can be well described by the linear spin-wave theory (LSWT), which was developed decades ago [1]. Upon reducing a magnetic system from three dimensions to two dimensions or one dimension, quantum fluctuations will be substantially enhanced, which consequently results in various intriguing and exotic physical phenomena [2]. The theoretical work by Bethe more than 80 years ago predicted that the $S=1 / 2$ antiferromagnetic Heisenberg chain cannot form a long-range magnetic ordering due to strong quantum fluctuations [3]. A domain-wall-like spin excitation called a "spinon" was proposed later, which has been experimentally confirmed in various real magnetic systems. Unfortunately, except for the $S=1 / 2$ spin-chain case, no exact analytical solution like Bethe's work has been developed for other one-dimensional (1D) antiferromagnetic Heisenberg spin systems. However, numerical methods were widely employed to study the ground states of more complicated 1D systems [4], such as 1D spin chains with $S>1 / 2$ and spin ladders with even/odd legs [5],

\footnotetext{
*guochu.deng@ansto.gov.au,

${ }^{\dagger}$ garry.mcintyre@ansto.gov.au
}

and successfully predicted their ground-state and dynamic behaviors.

As the simplest case of a 1D spin system, $\mathrm{Cu}^{2+}(S=1 / 2)$ spin chains are extensively investigated due to their close relationship with the cuprate high-temperature superconductors [5]. $\mathrm{CuO}_{2}$ chains can be divided into two classes, cornersharing spin chains and edge-sharing spin chains. The former are building blocks of cuprate superconductors and spin ladders. In corner-sharing $\mathrm{CuO}_{2}$ chains, the exchange interaction between the nearest neighbors $(\mathrm{NNs})$ comes from the superexchange through the $\sim 180^{\circ} \mathrm{Cu}-\mathrm{O}-\mathrm{Cu}$ pathway [6]. Such an interaction is experimentally demonstrated to be quite strong, ranging from 100 to $160 \mathrm{meV}$ [7]. In contrast, the $\mathrm{NN}$ exchange interaction along edge-sharing spin chains is much weaker due to the $\sim 90^{\circ} \mathrm{Cu}-\mathrm{O}-\mathrm{Cu}$ pathway [8-10]. The limited examples of edge-sharing cuprates share fewer common features due to the sensitivity of the interaction on the $\mathrm{Cu}-\mathrm{O}-\mathrm{Cu}$ bond angle [10]. Both ferromagnetic and antiferromagnetic interactions have been reported for this case [9,11-13].

$\mathrm{Sr}_{14-x} \mathrm{Ca}_{x} \mathrm{Cu}_{24} \mathrm{O}_{41}$ is a quasi-1D $S=1 / 2$ magnetic system, involving both corner-sharing and edge-sharing $\mathrm{CuO}_{2}$ spin chains [14]. The corner-sharing spin chains form two-leg spin ladders, as shown in Fig. 1(a). Therefore, this series of compounds can be considered to be a very good playground for studying the physics of these two different $1 \mathrm{D}$ and quasi1D magnetic systems. The other two special properties of 

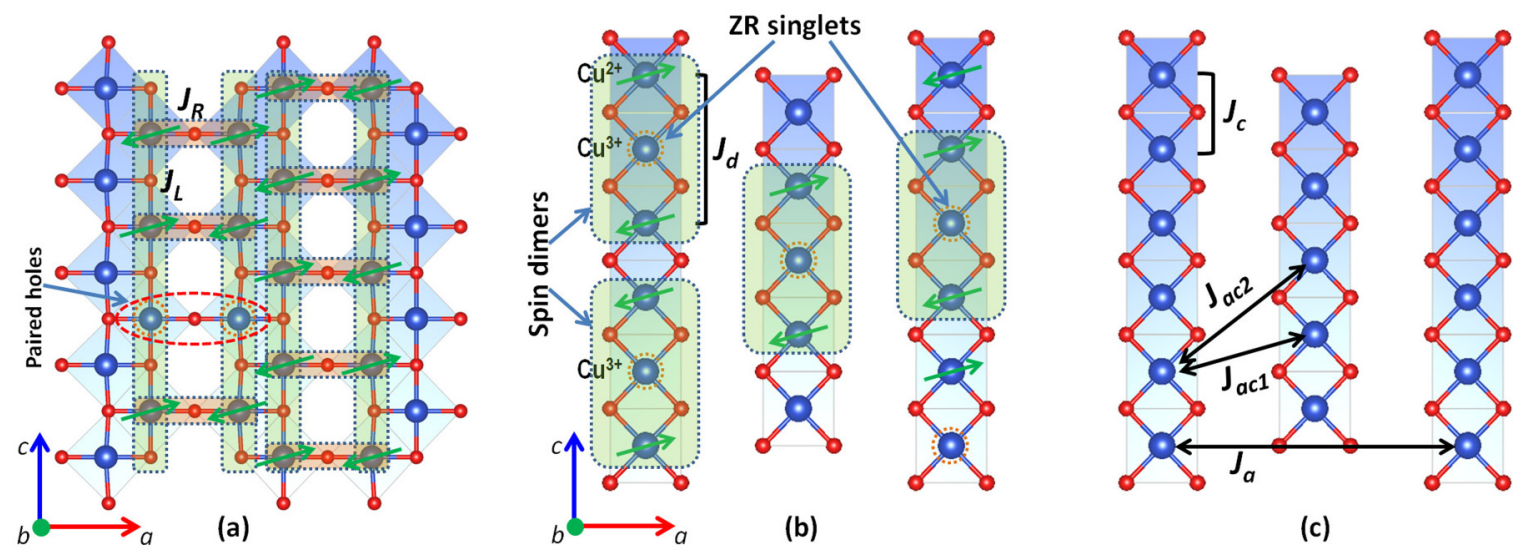

(c)

FIG. 1. (a) The spin-ladder sublattice of $\mathrm{Sr}_{14-x} \mathrm{Ca}_{x} \mathrm{Cu}_{24} \mathrm{O}_{41}$ consists of two-leg ladders, and $J_{\mathrm{R}}$ and $J_{\mathrm{L}}$ are exchange interactions along the rungs and the legs. Theory predicts that doped holes form pairs (see the red ellipse), which play important roles in its superconducting phase. (b) On the spin-chain sublattice of $\mathrm{Sr}_{14} \mathrm{Cu}_{24} \mathrm{O}_{41}$, so-called Zhang-Rice (ZR) singlets are formed by a copper hole hybridized with a hole in its neighboring oxygen. The two neighboring $\mathrm{Cu}^{2+}$ form spin dimers through the nonmagnetic $\mathrm{Cu}^{3+}$ [18]. (c) The holes in highly Ca-doped compounds were primarily driven to the spin-ladder sublattice due to the chemical pressure, which consequently leaves the spin chain nearly undoped. This magnetic system becomes long-range magnetic order at $4.2 \mathrm{~K} . J_{c}$ is the NN exchange interaction while $J_{a c 1}, J_{a c 2}$, and $J_{a}$ are possible interactions along the $a$ axis.

this series make it more attractive to physicists. First, this compound becomes superconductive under external pressure and by substituting $\mathrm{Ca}$ on the $\mathrm{Sr}$ site [15], bearing in mind that it has no $\mathrm{CuO}_{4}$ square plackets in this compound like other superconducting cuprates, but spin chains and spin ladders. Second, this system is intrinsically doped with holes, which can be driven back and forth between the chains and the ladders by simply controlling the doping level of $\mathrm{Ca}$ [14]. Therefore, one is not only able to study the superconducting mechanism of the spin ladder but also able to investigate the physics of hole-doped or undoped spin chains and ladders simply by controlling the Ca content. Furthermore, the relationships or interactions between these phenomena are more complicated topics to be addressed.

A recent study by some of the authors of the present paper showed how the incommensurate structures of the $\mathrm{Sr}_{14-x} \mathrm{Ca}_{x} \mathrm{Cu}_{24} \mathrm{O}_{41}$ series evolve with the Ca doping content, which provides direct structural evidence for the charge transfer from the spin chains to the ladders by increasing Ca doping level [14]. Our inelastic neutron-scattering experiments revealed that the increase of $\mathrm{Ca}$ doping did not close the spin gap of the spin ladder in all these compounds, but only suppressed its intensity due to the increase of hole doping [16]. In the highly doped compound $\mathrm{SrCa}_{13} \mathrm{Cu}_{24} \mathrm{O}_{41}$, long-range magnetic ordering has been observed and the coexistence of magnetic ordering and a spin-liquid ground state was demonstrated [17]. All these studies provide some answers to the interesting questions mentioned above. However, many open questions still remain.

In this paper, we investigate the low-energy excitation from the magnetic ordered phase in $\mathrm{SrCa}_{13} \mathrm{Cu}_{24} \mathrm{O}_{41}$ by inelastic neutron scattering. Considering the hole transfer from the chains to the ladder sublattice, this paper is a comparative research to the study of the hole-doped spin chain in the parent compound $\mathrm{Sr}_{14} \mathrm{Cu}_{24} \mathrm{O}_{41}$. In contrast to the singlet ground state of $\mathrm{Sr}_{14} \mathrm{Cu}_{24} \mathrm{O}_{41}$, the observed spin-wave excitation in $\mathrm{SrCa}_{13} \mathrm{Cu}_{24} \mathrm{O}_{41}$ demonstrates its two-dimensional (2D) feature rather than a 1D one in this compound. The physics of these two scenarios have been compared. The difference between the current compound and other edge-sharing cuprate systems has been addressed in order to provide a more general view of this special spin-chain system.

\section{SPIN-LADDER AND SPIN-CHAIN PHYSICS $\mathrm{OF} \mathrm{Sr}_{14-x} \mathrm{Ca}_{x} \mathrm{Cu}_{24} \mathrm{O}_{41}$}

The $\mathrm{Sr}_{14-x} \mathrm{Ca}_{x} \mathrm{Cu}_{24} \mathrm{O}_{41}$ series is formed by stacking the spin-ladder [Fig. 1(a)] and spin-chain [Figs. 1(b) and 1(c)] sublattices along the $b$ axis [14]. The ladder units and spinchain units are incommensurate along the $c$ axis. These two sublattices demonstrate quite different physics, such as spin dimerization [19], superconductivity [15], charge crystallization [20], charge transfer [14], and so on, which are remarkably changed by $\mathrm{Ca}$ doping, too. Here we will briefly introduce them separately in support of the further discussion in this paper.

\section{A. Spin ladder}

According to the theory [5], even-leg half-spin ladders are gapped spin systems no matter the ratio of $J_{\mathrm{R}} / J_{\mathrm{L}}$, where $J_{\mathrm{R}}$ is the interaction along the rungs and $J_{\mathrm{L}}$ is the interaction along the legs [see Fig. 1(a)]. In the case of $J_{\mathrm{R}} / J_{\mathrm{L}} \gg 1$, the spins on each rung form a singlet dimer, which is the ground state of an even-leg spin-ladder system. As proposed by Dagotto and Rice [5], by lightly doping an $S=1 / 2$ even-leg spin-ladder system with holes, one could expect $d$-wave superconductivity with a spin gap in this ladder due to hole pairing along the rungs, as shown in Fig. 1(a). Such superconductivity is very similar to what is observed in $2 \mathrm{D}$ cuprates. Both of them possess a spin gap and $d_{x^{2}+y^{2}}$ symmetry. From this point of view, disclosing the superconducting mechanism in spin ladders undoubtedly helps to improve our understanding of the superconductivity in $2 \mathrm{D}$ cuprates [5]. 
The spin ladder in $\mathrm{Sr}_{14-x} \mathrm{Ca}_{x} \mathrm{Cu}_{24} \mathrm{O}_{41}$ attracts much more attention than the spin chain because the former was suggested to be the platform of the observed superconductivity in the highly Ca-doped compounds [21]. In the parent compound, $\mathrm{Sr}_{14} \mathrm{Cu}_{24} \mathrm{O}_{41}$, a strong spin-gap excitation was observed by using inelastic neutron scattering [16,22], indicating $J_{\mathrm{L}}>J_{\mathrm{R}}$ in the parent compound. Correspondingly, no superconductivity was observed under external pressure up to $6.5 \mathrm{GPa}$ [23], in contrast to the superconductivity of highly Ca-doped compounds at $\sim 3 \mathrm{GPa}$ [23] or even lower uniaxial pressure [24]. So far, there is no solid theoretical understanding of why the isovalent doping with $\mathrm{Ca}$ changes the superconductivity of this series of compounds.

Ca doping extensively changes the physics of the ladder sublattice, which may induce superconductivity theoretically predicted. First, holes transfer to the ladder sublattice, which makes hole pairing possible as described in the theory. Second, we confirmed that the spin gap, which is the driving force for the hole pairing, still exists at $\sim 32.5 \mathrm{meV}$ in highly Cadoped compounds [17]. The high pressure could play another critical role in changing the ratio $J_{\mathrm{R}} / J_{\mathrm{L}}$ from less than 1 to larger than 1 by distorting the lattice even though our previous measurement of the spin gap under a hydrostatic pressure of 2 $\mathrm{GPa}$ was inconclusive due to the challenges of the experiments [25]. These facts indicate that $\mathrm{Ca}$ doping plays a critical role in the superconductivity in this spin-ladder system, just as the theory predicted.

\section{B. Spin chain}

The spin-chain sublattice of $\mathrm{Sr}_{14-x} \mathrm{Ca}_{x} \mathrm{Cu}_{24} \mathrm{O}_{41}$ consists of edge-sharing $\mathrm{CuO}_{2}$ squares, as shown in Figs. 1(b) and 1(c), which are separated from the spin ladder by a layer of alkaline-earth cations [14]. In the parent compound, the spin chains look like wrinkled ribbons running along the $c$ axis, in which the $\mathrm{Cu}-\mathrm{O}$ bond length varies over a quite large range due to the incommensurate modulation of the crystal structure. Such a modulation is strongly suppressed in the highly Ca-doped compounds. The wrinkles on the spin chain seem to be "ironed" into much flatter ones compared with those in the parent compound [14].

Doped holes are primarily located on the spin-chain sublattice in $\mathrm{Sr}_{14} \mathrm{Cu}_{24} \mathrm{O}_{41}$, which fundamentally changes the chain physics. Doped holes combine with $\mathrm{Cu}^{2+}$ ions to form nonmagnetic $\mathrm{Cu}^{3+}$ ions, becoming so-called Zhang-Rice singlets. Two neighboring $\mathrm{Cu}^{2+}$ spins of each $\mathrm{Cu}^{3+}$ ion form a singlet dimer [26] at low temperature [see Fig. 1(b)], in which the two $\mathrm{Cu}^{2+}$ spins couple antiferromagnetically [18]. Consequently, such a spin-chain system undergoes a singlet dimer ground state [18], which is a kind of spin-liquid state with short-range interactions but no long-range magnetic ordering. The chain contribution to the susceptibility shows a broad peak at $\sim 80 \mathrm{~K}$ [19], which corresponds to the singlet-triplet excitation of the spin dimers.

Increasing Ca content in $\mathrm{Sr}_{14-x} \mathrm{Ca}_{x} \mathrm{Cu}_{24} \mathrm{O}_{41}$ substantially reduces the number of holes in the chains [16], suppressing the Zhang-Rice singlets and the spin-liquid state [14]. The physics of the spin chains in highly Ca-doped $\mathrm{Sr}_{14-x} \mathrm{Ca}_{x} \mathrm{Cu}_{24} \mathrm{O}_{41}$ have not been investigated yet. Considering the large spacings between each chain along both the $a$ and $b$ directions, the chains in $\mathrm{SrCa}_{13} \mathrm{Cu}_{24} \mathrm{O}_{41}$ seem to constitute an ideal 1D spin system, which becomes an interesting playground for testing low-dimensional quantum effects. By studying the long-range magnetic ordering in $\mathrm{SrCa}_{13} \mathrm{Cu}_{24} \mathrm{O}_{41}$ below $4.2 \mathrm{~K}$, alongside strong magnetic excitation, we are seeking deep insights into the low-dimensional physics of the spin-chain system in this compound. Therefore, we performed inelastic neutronscattering experiments on a large high-quality single crystal of $\mathrm{SrCa}_{13} \mathrm{Cu}_{24} \mathrm{O}_{41}$ and determined the spin-wave dispersion and hence the magnetic exchange interaction along the different directions.

\section{EXPERIMENT}

The single-crystal sample of $\mathrm{SrCa}_{13} \mathrm{Cu}_{24} \mathrm{O}_{41}$ for the inelastic neutron-scattering experiment was grown by using the travelling-solvent floating-zone technique in an imaging furnace with a high oxygen pressure ( 32 bars) [27]. For the experiment on the time-of-flight (TOF) spectrometer PELICAN [28] at Open Pool Australian Lightwater reactor (OPAL), the single crystal was aligned in the $b c$ plane using the neutron Laue camera JOEY [29]. An incident neutron beam with a wavelength of $4.69 \AA(3.75 \mathrm{meV})$ was used for this experiment. The energy resolution for this configuration on PELICAN is about $0.135 \mathrm{meV}$. The sample was rotated over a range of $110^{\circ}$ with $1^{\circ}$ per step. The experimental data were processed into the scattering function $S(q, \omega)$ by using the software package HORACE from ISIS [30]. The cold-neutron triple-axis spectrometer SIKA [31] at OPAL was used to measure the spin-wave dispersion along the $Q_{\mathrm{H}}, Q_{\mathrm{K}}$, and $Q_{\mathrm{L}}$ directions within the $a c$ and $b c$ scattering planes. A double-focusing monochromator and open-open- $60^{\prime}-60^{\prime}$ collimation with a fixed final energy of $E_{f}=5 \mathrm{meV}$ were used for the experiments on SIKA. A cooled Be filter was utilized on the scattering side of the instrument in order to eliminate second-order contamination. The energy resolution of this configuration is about $0.13 \mathrm{meV}$ at the elastic line and about $0.16 \mathrm{meV}$ at the energy transfer of $4 \mathrm{meV}$. The data collected from SIKA were fitted to a double Lorentzian cross section by convoluting with the instrumental resolution.

\section{RESULTS AND DISCUSSIONS}

The TOF data of $\mathrm{SrCa}_{13} \mathrm{Cu}_{24} \mathrm{O}_{41}$ at $1.5 \mathrm{~K}$ are presented in Fig. 2. Figures 2(a) and 2(b) show the energy excitation in an energy window from 0.5 to $2.0 \mathrm{meV}$ in the $Q_{\mathrm{K}}-Q_{\mathrm{L}}$ plane and the dispersion along the $Q_{\mathrm{L}}$ direction in the energy range from 0 to $2.5 \mathrm{meV}$, respectively. These two figures are directly cut from the 3D plot shown in Fig. 2(c). From the 3D plot, we can clearly identify the inelastic excitation running along all $Q_{\mathrm{K}}$ at $Q_{\mathrm{L}}=3$ r.l.u. (reciprocal lattice unit), which corresponds to the horizontal stripe of high intensity at $Q_{\mathrm{L}}=$ 3 r.l.u. in Fig. 2(a). This stripy excitation should be attributed to its nondispersive feature along the $Q_{\mathrm{K}}$ direction. Viewing in the $Q_{\mathrm{L}}-E$ plane in Fig. 2(b), the apparent broadening of this excitation peak at higher energy transfer indicates its dispersion along the $Q_{\mathrm{L}}$ direction. Further cuts at various energy transfers of Fig. 2(b) generate the constant energy curves shown in Fig. 2(d). The fitting to these curves indicates that the excitation peak gradually splits into two peaks when 



FIG. 2. The low-energy magnetic excitation of $\mathrm{SrCa}_{13} \mathrm{Cu}_{24} \mathrm{O}_{41}$ measured on PELICAN at $1.5 \mathrm{~K}$. (a) The constant energy cut in the $Q_{\mathrm{K}}-$ $Q_{\mathrm{L}}$ plane by integrating intensity over the energy range from 0.5 to $2 \mathrm{meV}$. (b) The magnetic excitation in the $Q_{\mathrm{L}}-E$ plane with the integrated intensity over the $Q_{\mathrm{K}}$ range from -5 to 0 r.l.u. (c) The $3 \mathrm{D}$ view of the magnetic excitation in the $Q_{\mathrm{K}}-Q_{\mathrm{L}}-E$ space. (d) The constant energy cuts at 1, 1.5, and $2 \mathrm{meV}$ from panel (b) with a $1-\mathrm{meV}$ window. The curves are intentionally shifted along the intensity axis in order to avoid overlapping.

increasing the energy, which confirms the dispersion of this excitation along $Q_{\mathrm{L}}$. All these excitation features disappeared at a raised temperature of $50 \mathrm{~K}$ (not shown here) except the strong signal observed around (040) [see Fig. 2(a)]. Therefore, we safely attribute the stripy excitation to the spin-wave excitation of the magnetic ordered state, but we attribute the excitation near (040) to the phonon excitation. Due to the limited energy-transfer range covered by PELICAN at this configuration, the top of the excitation could not be observed. Within the good energy resolution $(\sim 0.135 \mathrm{meV})$ of the current setup, the excitation did not show any energy gap at the zone center, suggesting a gapless excitation.

In order to measure the magnetic excitation in detail, we carried out inelastic neutron-scattering experiments on the cold-neutron triple-axis spectrometer SIKA with a fixed final energy of $5 \mathrm{meV}$. The results are presented in Figs. 3-5. The spin chains in $\mathrm{SrCa}_{13} \mathrm{Cu}_{24} \mathrm{O}_{41}$ run along the $c$ axis of the crystal. The interaction along this direction is supposed to be the strongest and most important for the determination of the dynamic behavior of the spin chains. Therefore, we present the dispersion of the magnetic excitation along the $c$ axis first. Figure 3(a) clearly shows an intense excitation peak measured along the $Q_{\mathrm{L}}$ direction with a constant energy transfer of $0.5 \mathrm{meV}$ at $1.5 \mathrm{~K}$. The peak completely disappeared at $100 \mathrm{~K}$, which confirms its magnetic origin. On increasing the energy transfer, the central peak gradually broadens and splits into two peaks while the intensity drops. Above an energy transfer of $2 \mathrm{meV}$, the two peaks are clearly resolved and well separated. This result is consistent with the constant energy cut at $2 \mathrm{meV}$ from PELICAN in Fig. 2(d). The asymmetrical shapes of the two peaks are not only due to the instrument resolution but also due to the inclined background at small scattering angles. The background increases at higher energy transfers. Near the zone boundary, an energy scan was conducted with an extended counting time. Figure 3(f) shows an energy scan at a fixed $\boldsymbol{Q}$ point of $(0,1,3.9)$, i.e., close to the boundary of the Brillouin zone. A peak at $5.3 \pm 0.2 \mathrm{meV}$ was observed. The data were fitted by convoluting with the instrument resolution.

The spin chains in $\mathrm{SrCa}_{13} \mathrm{Cu}_{24} \mathrm{O}_{41}$ are stacked along the $b$ axis with the alkaline-earth cations and spinladder planes in between. We expected that the interaction 

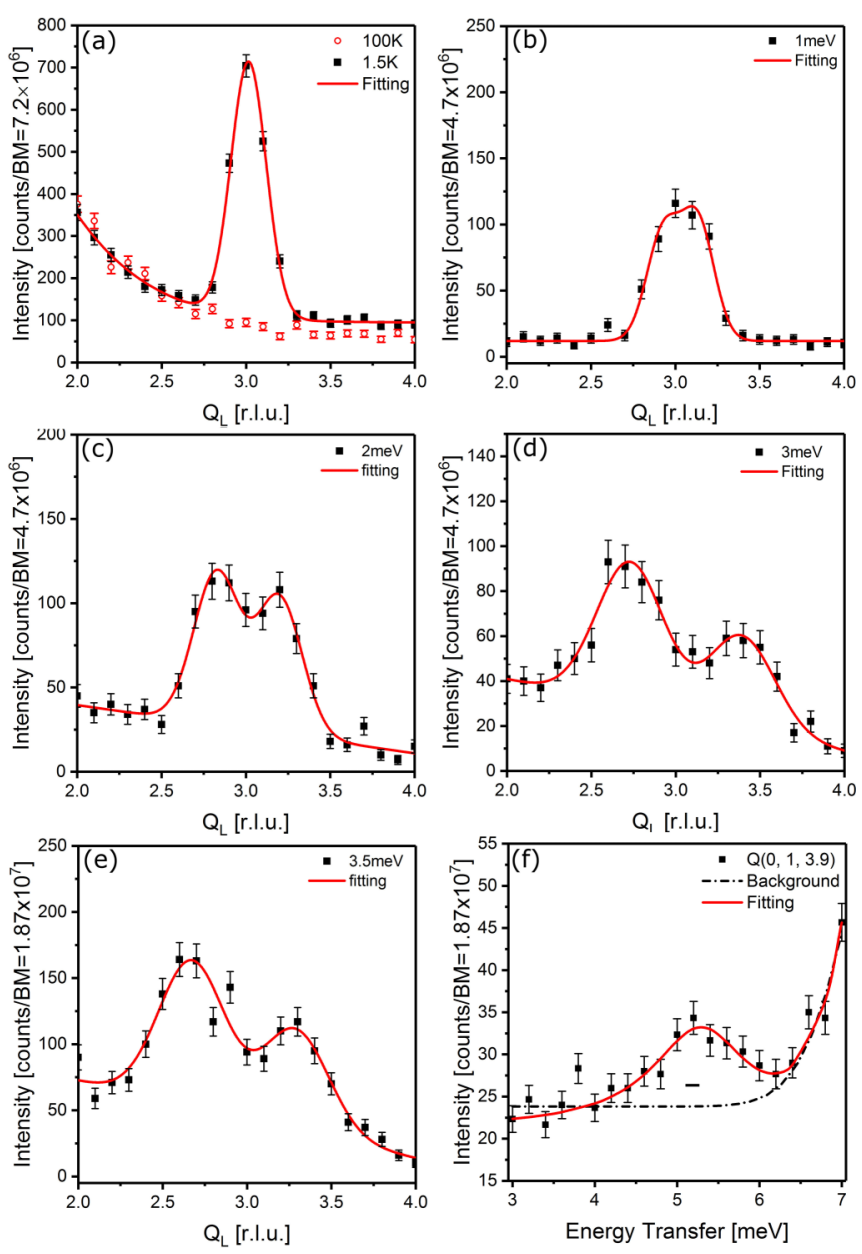

FIG. 3. $Q_{\mathrm{L}}$ scans at energy transfers of $0.5 \mathrm{meV}$ (a), $1 \mathrm{meV}$ (b), $2 \mathrm{meV}$ (c), $3 \mathrm{meV}$ (d), and $3.5 \mathrm{meV}$ (e) through the antiferromagnetic zone center $\boldsymbol{Q}(0,1,3)$ at $1.5 \mathrm{~K}$ with a fixed- $E_{f}$ mode on SIKA. (f) An energy scan at $\boldsymbol{Q}(0,1,3.9)$, i.e., close to the boundary of the Brillouin zone, with a fixed- $E_{f}$ mode on SIKA. The solid lines are fitted to the double-Lorentzian cross section convoluted with the instrumental resolution, where the background is shown by the dash-dotted line. The horizontal bar below the peak in panel (f) shows the instrumental resolution at the energy transfer of $4 \mathrm{meV}$.

along the $b$ axis is very weak. In order to confirm this speculation, the measurements at different $Q_{\mathrm{K}}$ positions were carried out on SIKA and the results are shown in Fig. 4. The three $Q_{\mathrm{L}}$ scans at the different $Q_{\mathrm{K}}$ values $(=0.8,1.2$, 1.5 r.l.u.) in Figs. 4(a)-4(c) basically show similar excitation peaks at the energy transfer of $0.5 \mathrm{meV}$. Figure 4(d) shows the comparison of the intensity of the peak at $Q_{\mathrm{L}}=3$ r.l.u. and the background intensity at an off-peak position $Q_{\mathrm{L}}=2.5$ r.l.u. The intensity of the peak at lower $Q_{\mathrm{K}}$ is slightly higher due to the decrease of the magnetic form factor at higher $\boldsymbol{Q}$ values. The data indicate that there is no dispersion along the $Q_{\mathrm{K}}$ direction. These results agree well with the stripy feature along the $Q_{\mathrm{K}}$ direction observed on PELICAN [see Figs. 2(a) and $2(\mathrm{c})]$.

The spin chains lie in the $a c$ plane, in which the interaction between the neighbor chains is another important dynamic factor which determines the dimensionality of this spin-chain system. Our inelastic neutron-scattering experiment on SIKA was performed in the vicinity of $\boldsymbol{Q}(0,0,3)$ in the $a c$ scattering plane. Selected results are shown in Fig. 5. Constant energy scans are plotted in Figs. 5(a)-5(c) for energy transfers of $0.5,1.5$, and $2.5 \mathrm{meV}$, respectively. The peak splitting at the larger energy transfer clearly indicates the dispersion along the $Q_{\mathrm{H}}$ direction. An energy scan was carried out at the boundary of the Brillouin zone at $\boldsymbol{Q}(1,0,3)$ and is shown in Fig. 5(d). The excitation was observed at $4.2 \pm 0.2 \mathrm{meV}$, which is lower than the zone boundary energy along the $Q_{\mathrm{L}}$ direction. All the collected experimental data for the constant energy and constant $\boldsymbol{Q}$ scans for the directions $Q_{\mathrm{H}}$ and $Q_{\mathrm{L}}$ are summarized in the dispersion curve shown in Fig. 6.

Both the spin-ladder and spin-chain subsystems in $\mathrm{SrCa}_{13} \mathrm{Cu}_{24} \mathrm{O}_{41}$ are quasi-1D or 1D magnetic systems. Our previous inelastic neutron-scattering experiment confirmed the spin-gap excitation at $\sim 32 \mathrm{meV}$ in this compound $[16,17]$. This excitation was attributed to the spin ladder due to its consistency with most other results reported for the spin ladders in the parent compound [22,32]. The exchange interactions along the legs and rungs were estimated to be $\sim 130$ and $\sim 72 \mathrm{meV}$ [22], respectively, or larger, which agrees well with the antiferromagnetic interactions through the $180^{\circ} \mathrm{Cu}-\mathrm{O}$ $\mathrm{Cu}$ pathway in cuprates with square lattices [33]. Therefore, we attribute the low-energy excitation observed above to the spin-chain sublattice. As reported in other edge-sharing $\mathrm{CuO}_{2}$ chain systems, such as $\mathrm{CuGeO}_{3}$ [34], the exchange interactions between neighboring $\mathrm{Cu}^{2+}$ ions through a $90^{\circ}$ $\mathrm{Cu}-\mathrm{O}-\mathrm{Cu}$ pathway are much weaker than the excitation in the corner-sharing $\mathrm{CuO}_{2}$ systems with a $180^{\circ} \mathrm{Cu}-\mathrm{O}-\mathrm{Cu}$ pathway.

As described above and shown in Fig. 1, the spin chains in $\mathrm{SrCa}_{13} \mathrm{Cu}_{24} \mathrm{O}_{41}$ are built by edge-sharing $\mathrm{CuO}_{2}$ squares. Comparing with a corner-sharing spin chain, an edge-sharing spin chain has much weaker interaction $\left(J_{c}\right)$ within the chains. Along the stacking direction, namely, the $b$ axis, the interaction is very weak and negligible due to the large interlayer spacing, which is confirmed by the dispersionless behavior of the spin excitation as shown above. Similar weak interlayer interactions have been reported in $\mathrm{La}_{2} \mathrm{CuO}_{4}$ [33] and $\mathrm{Rb}_{2} \mathrm{MnF}_{4}$ [35], both of which were classified as $2 \mathrm{D}$ magnets. Considering the intralayer interactions, there are several possible interactions, such as $J_{c}, J_{a c 1}, J_{a c 2}$, and $J_{a}$, as shown in Fig. 1(c). Ignoring the single-ion anisotropy and taking in-plane intrachain and interchain interactions into account, the Heisenberg Hamiltonian of the spin-chain system in $\mathrm{SrCa}_{13} \mathrm{Cu}_{24} \mathrm{O}_{41}$ reads as follows:

$$
H=\sum_{i, j} J_{i j} \overrightarrow{\boldsymbol{S}}_{i} \cdot \overrightarrow{\boldsymbol{S}}_{j}
$$

where $J_{i j}$ includes $J_{c}, J_{a c 1}, J_{a c 2}$, and $J_{a}$ in Fig. 1(c). According to LSWT, the dispersion relationship could be derived in the following formula [36]:

$$
\begin{aligned}
h \omega= & S\left(\left\{J_{c}\left[\cos \left(q_{\mathrm{L}}\right)-1\right]+J_{a}\left[\cos \left(q_{\mathrm{H}}\right)-1\right]+2 J_{a c 1}\right.\right. \\
& \left.+2 J_{a c 2}\right\}^{2}-\left[2 J_{a c 1} \cos \left(\frac{q_{\mathrm{H}}}{2}\right) \cos \left(\frac{q_{\mathrm{L}}}{2}\right)\right. \\
& \left.\left.+2 J_{a c 2} \cos \left(\frac{q_{\mathrm{H}}}{2}\right) \cos \left(\frac{3 q_{\mathrm{L}}}{2}\right)\right]^{2}\right)^{1 / 2}
\end{aligned}
$$



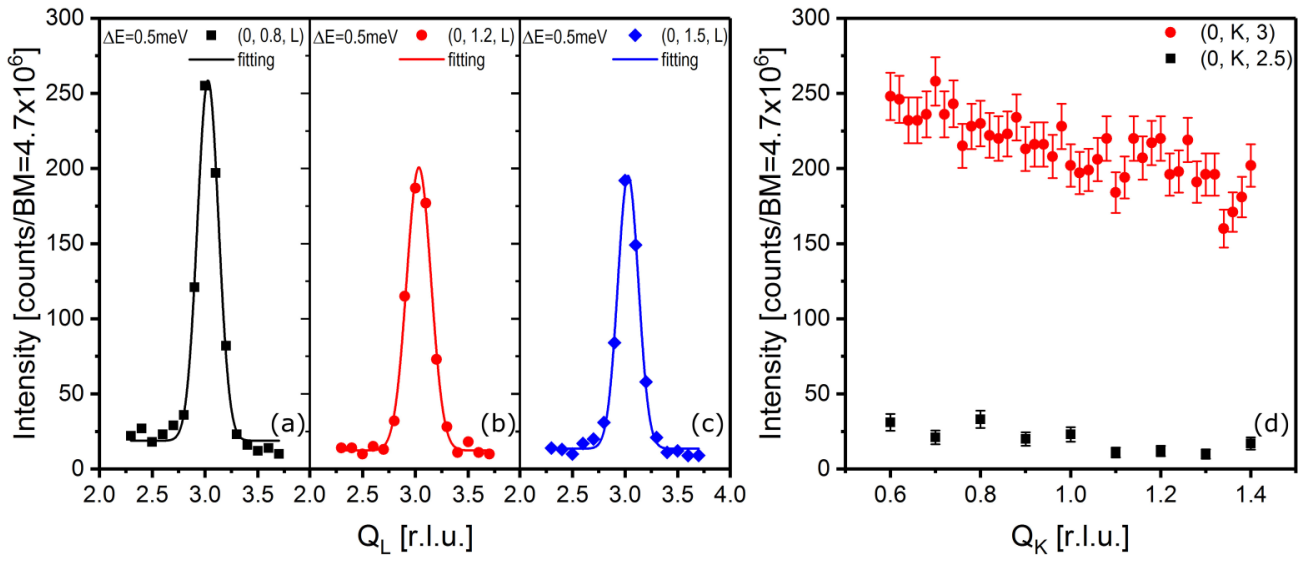

FIG. 4. $Q_{\mathrm{L}}$ scans at the energy transfer $0.5 \mathrm{meV}$ with $Q_{\mathrm{K}}=0.8(\mathrm{a}), Q_{\mathrm{K}}=1.0(\mathrm{~b})$, and $Q_{\mathrm{K}}=1.2$ (c). (d) The $Q_{\mathrm{K}}$ scans at the peak position with $Q_{\mathrm{L}}=3$ and off-peak position at $Q_{\mathrm{L}}=2.5$.

where $S$ is the moment size, and $q_{\mathrm{H}}$ and $q_{\mathrm{L}}$ correspond to the reciprocal-lattice units along the $a^{*}$ and $c^{*}$ directions.

We have tried many different combinations of the different exchange interactions in the model above to fit the experimental data. Finally, it was found that the experimental data cannot be well described if $J_{a c 1}$ and $J_{a c 2}$ are in the comparable range as $J_{c}$ and $J_{a}$. By ignoring these two exchange interactions from the model above, therefore, we are able to fit our data well to the model, which gives $J_{c}=5.4 \pm 0.1 \mathrm{meV}$ and $J_{a}=$ $4.4 \pm 0.3 \mathrm{meV}$. The $\mathrm{NN}$ intrachain interaction is antiferro-
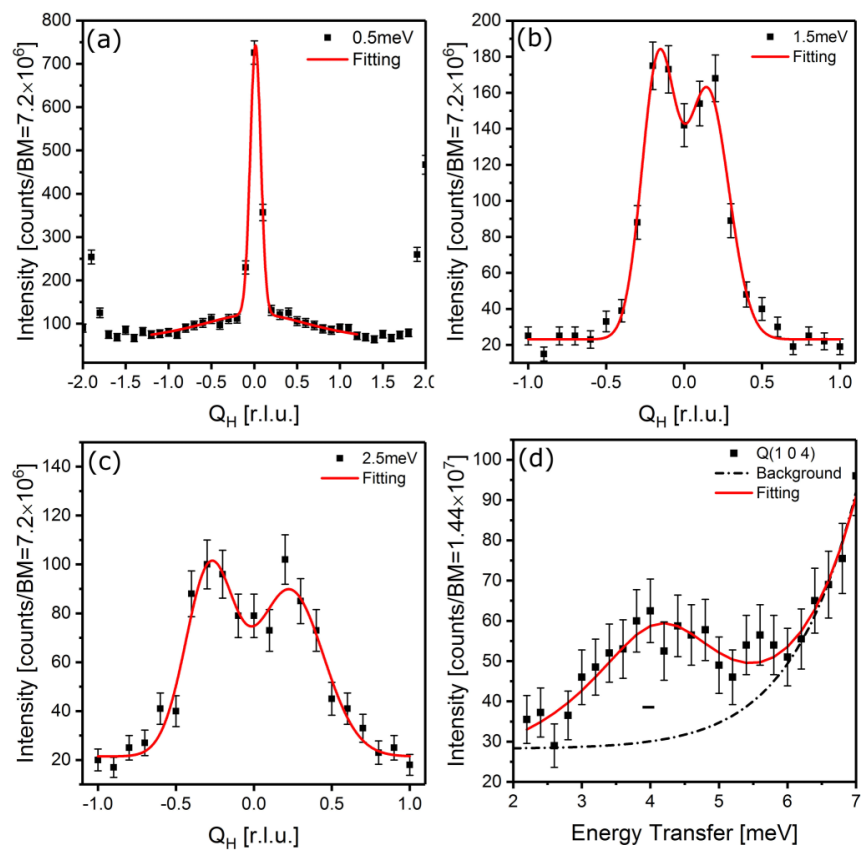

FIG. 5. $Q_{\mathrm{K}}$ scans at energy transfers of $0.5 \mathrm{meV}$ (a), $1.5 \mathrm{meV}$ (b), and $2.5 \mathrm{meV}$ (c) through $\boldsymbol{Q}(0,0,3)$ with a fixed- $E_{f}$ mode on SIKA. (d) An energy scan at the boundary of the Brillouin zone at $\boldsymbol{Q}(1,0,3)$ with a fixed- $E_{f}$ mode on SIKA. The solid lines are fitted to the double-Lorentzian cross section convoluted with the instrumental resolution. The background is shown by the dash-dotted line. The horizontal bar below the excitation peak in panel (d) shows the instrumental resolution at the energy transfer of $4 \mathrm{meV}$. magnetic, which was confirmed by the linear relationship of the dispersion curve observed on both PELICAN and SIKA in the low $-q_{\mathrm{L}}$ range. Figures $6(\mathrm{a})$ and $6(\mathrm{~b})$ show the fitting to the experimental data along the $Q_{\mathrm{L}}$ and $Q_{\mathrm{H}}$ directions with the LSWT model. The fitted dispersion relations are quite consistent with the experimental results.

In comparison with the 2D magnetic systems, such as $\mathrm{La}_{2} \mathrm{CuO}_{4}$ [33] and $\mathrm{Rb}_{2} \mathrm{MnO}_{4}$ [35], the spin-chain system in $\mathrm{SrCa}_{13} \mathrm{Cu}_{24} \mathrm{O}_{41}$ has a similar $2 \mathrm{D}$ spin-wave feature. According to the literature, the interplane interaction is several orders of magnitude weaker than the intraplane interaction in $\mathrm{La}_{2} \mathrm{CuO}_{4}$ and $\mathrm{Rb}_{2} \mathrm{MnO}_{4}$, which causes the nondispersive spin-wave excitation along the stacking direction of layers. However, such a relatively weak interaction is strong enough to drive these two systems into long-range magnetic ordering states. For example, $\mathrm{Rb}_{2} \mathrm{MnO}_{4}$ orders into the antiferromagnetic phase at $38.4 \mathrm{~K}$ [35]. In contrast, $\mathrm{SrCa}_{13} \mathrm{Cu}_{24} \mathrm{O}_{41}$ becomes antiferromagnetic ordering at $4.2 \mathrm{~K}$ [17], indicating a much weaker interplane interaction.

It is interesting to compare the current spin-chain system with other edge-sharing spin chains, such as $\mathrm{CuGeO}_{3}[11,34]$, $\mathrm{Ca}_{2} \mathrm{Y}_{2} \mathrm{Cu}_{5} \mathrm{O}_{10}[12,36]$, and $\mathrm{Li}_{2} \mathrm{CuO}_{2}$ [13,37]. $\mathrm{CuGeO}_{3}$ has similar spin chains as the current spin-chain system and
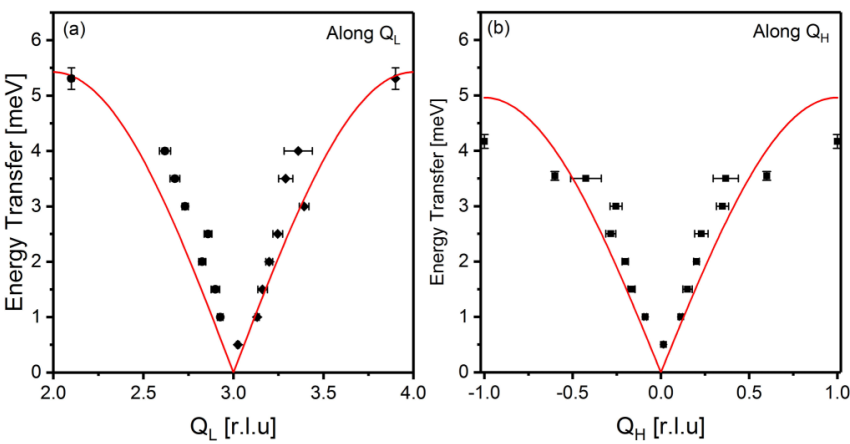

FIG. 6. The dispersion curves along the $Q_{\mathrm{L}}$ (a) and $Q_{\mathrm{H}}$ (b) directions. The black dots are experimental data while the red curves are the dispersion relations as obtained by fitting to the right branches of the experimental data while the left branches are plotted symmetrically with the same fitted parameters. 
undergoes a spin-Peierls phase transition at $14 \mathrm{~K}$, where the $\mathrm{Cu}^{2+}$ spins form a spin-dimer ground state. Inelastic neutron scattering reveals that the nearest-neighbor exchange $J_{c}$ is antiferromagnetic and around $10.4 \mathrm{meV}$. The nearest intrachain interaction of $\mathrm{Y}_{2} \mathrm{Ca}_{2} \mathrm{Cu}_{5} \mathrm{O}_{10}$ is ferromagnetic $(\sim-8 \mathrm{meV})$. The chain in $\mathrm{Li}_{2} \mathrm{CuO}_{2}$ is also ferromagnetic with a nearest intrachain interaction of $\sim-14.7 \mathrm{meV}$, reported by a recent study [13]. In the previous study on the similar compound, $\mathrm{La}_{5} \mathrm{Ca}_{9} \mathrm{Cu}_{24} \mathrm{O}_{41}$ [9], it was found that its $\mathrm{NN}$ exchange interaction is weak ferromagnetic $(-0.2 \mathrm{meV})$. However, the magnetic phase orders at about $10 \mathrm{~K}$, which is not consistent with the weak antiferromagnetic interaction. From the comparison of all these studies, we conclude that the $\mathrm{NN}$ intrachain interactions of these chain systems vary dramatically. The variation of the $\mathrm{Cu}-\mathrm{O}-\mathrm{Cu}$ bond angles and lengths could be the main reason. According to the Goodenough-Kanamori-Anderson rule [38], when the $\mathrm{Cu}-\mathrm{O}-\mathrm{Cu}$ bond angle changes from $180^{\circ}$ towards $90^{\circ}$, the exchange interaction evolves from a positive (antiferromagnetic) value to a negative (ferromagnetic) value. According to our previous study [14], the variation of the bond angle $\mathrm{Cu}-\mathrm{O}-\mathrm{Cu}$ significantly increases with the increase of $\mathrm{Ca}$ doping, in which the maximum angle could reach $96^{\circ}$ due to the structural modulation of this incommensurate system. Such variation may be even larger in $\mathrm{SrCa}_{13} \mathrm{Cu}_{24} \mathrm{O}_{41}$. Such a bond-angle variation could be the main reason for the antiferromagnetic interaction observed in this compound, which is evidenced by the sharp linear dispersion at the low- $q_{\mathrm{L}}$ range seen in the PELICAN data.

Since the parent compounds $\mathrm{Sr}_{14} \mathrm{Cu}_{24} \mathrm{O}_{41}$ and $\mathrm{SrCa}_{13} \mathrm{Cu}_{24} \mathrm{O}_{41}$ have very similar crystal structures, one may expect that they should have similar dynamic behavior from their spin-chain sublattices. Actually, the previous and current experimental results indicate that this is not the case. Matsuda et al. [18] have measured the magnetic excitation from the spin-chain sublattice of $\mathrm{Sr}_{14} \mathrm{Cu}_{24} \mathrm{O}_{41}$. They observed that the excitation ranges from 9 to $11 \mathrm{meV}$ at low temperature, showing very little dispersion along the chain. This observation was explained by the spin-dimer model for the chains. As mentioned above, the spin-chain sublattice in $\mathrm{Sr}_{14} \mathrm{Cu}_{24} \mathrm{O}_{41}$ is highly doped with holes. There are about 5.5 holes on ten $\mathrm{CuO}_{2}$ square units. At low temperature, these holes localize to $\mathrm{Cu}^{2+}$ ions to form Zhang-Rice singlets [26]. The two neighboring $\mathrm{Cu}^{2+}$ ions of each Zhang-Rice singlet form a spin-down and spin-up singlet dimer state. Due to the large number of the holes in the chains, these singlet dimers dominate the physics of the spin-chain sublattice of $\mathrm{Sr}_{14} \mathrm{Cu}_{24} \mathrm{O}_{41}$ at low temperature. The magnetic excitation observed at $\sim 10 \mathrm{meV}$ corresponds to the singlet-to-triplet excitation of these spin dimers, which explains the spin gap and the broad peak at $\sim 80 \mathrm{~K}$ in the susceptibility measurement [39].
The excitation scenario is completely different in $\mathrm{SrCa}_{13} \mathrm{Cu}_{24} \mathrm{O}_{41}$. Due to the high Ca-doping level, the holes are mainly driven to the spin-ladder sublattice in $\mathrm{SrCa}_{13} \mathrm{Cu}_{24} \mathrm{O}_{41}$ [14]. The spin chain in this compound, therefore, becomes a "non-hole-doped" $\mathrm{Cu}^{2+} \mathrm{O}_{2}$ spin chain. Due to the magnetic ordering, the excitation from the spin chains in this compound is either a spin-wave type or a spinon type of excitation. The above analysis of the inelastic neutron-scattering data has confirmed the 2D spin-wave excitation in $\mathrm{SrCa}_{13} \mathrm{Cu}_{24} \mathrm{O}_{41}$. The major difference of the chain dynamics in $\mathrm{SrCa}_{13} \mathrm{Cu}_{24} \mathrm{O}_{41}$ and $\mathrm{Sr}_{14} \mathrm{Cu}_{24} \mathrm{O}_{41}$ is that the former has a gapless excitation while the latter has a gapped excitation. The former one propagates in the magnetic lattice of the spin chain, especially along the chain, while the latter is nearly localized.

In conclusion, we studied the low-energy excitation spectrum from the magnetic ordered state of $\mathrm{SrCa}_{13} \mathrm{Cu}_{24} \mathrm{O}_{41}$. The excitation shows dispersions along the $c$ and $a$ directions but is nondispersive along the $b$ direction, indicating a 2D magnetic nature. The excitations were confirmed to originate from the spin-chain sublattice in this compound. Fitting to the experimental data to LSWT, the nearest-neighbor interaction of the chain was found to be $5.4 \mathrm{meV}$ and antiferromagnetic, which is comparable with the value observed in $\mathrm{CuGeO}_{3}$. The current magnet is different from the spin chain in the parent compound $\mathrm{Sr}_{14} \mathrm{Cu}_{24} \mathrm{O}_{41}$ because the chain physics of $\mathrm{Sr}_{14} \mathrm{Cu}_{24} \mathrm{O}_{41}$ is dominated by the spin dimers around ZhangRice singlets. The spin chains of $\mathrm{SrCa}_{13} \mathrm{Cu}_{24} \mathrm{O}_{41}$ are continuous and free of doped holes due to holes transferring to the ladder sublattice. This fact explains the spin-gap excitation in $\mathrm{Sr}_{14} \mathrm{Cu}_{24} \mathrm{O}_{41}$ but the gapless excitation for $\mathrm{SrCa}_{13} \mathrm{Cu}_{24} \mathrm{O}_{41}$ in this paper. The interaction between the spin-chain and spinladder systems in the magnetic ordered state of this magnet is still an open question for further study in the future.

\section{ACKNOWLEDGMENTS}

The authors thank ANSTO for the allocation of neutron beam time on SIKA and PELICAN (Grants No. P5715, No. P5214, and No. P4335). G.D. and H.L. acknowledge support from the National Natural Science Foundation of China (Grant No. 11674372). H.L. and S.L. are also supported by the National Key Research and Development Program of China (Grants No. 2017YFA0302903, No. 2017YFA0303103, and No. 2016YFA0300502), the Strategic Priority Research Program (B) of the Chinese Academy of Sciences (Grant No. XDB07020300), and the Youth Innovation Promotion Association of Chinese Academy of Sciences (Grant No. 2016004). W.R. and S.C. acknowledge support from the National Natural Science Foundation of China (Grants No. 11774217, No. 51372149, and No. 51672171) and the National Key Basic Research Program of China (Grant No. 2015CB921600).
[1] T. Holstein and H. Primakoff, Phys. Rev. 58, 1098 (1940).

[2] H. Beck, M. W. Puga, and G. Müller, J. Appl. Phys. 52, 1998 (1981).

[3] H. Bethe, Z. Phys. 71, 205 (1931).

[4] J. d. Cloizeaux and J. J. Pearson, Phys. Rev. 128, 2131 (1962).

[5] E. Dagotto and T. M. Rice, Science 271, 618 (1996).
[6] S. K. Satija, J. D. Axe, G. Shirane, H. Yoshizawa, and K. Hirakawa, Phys. Rev. B 21, 2001 (1980).

[7] X. Rocquefelte, K. Schwarz, and P. Blaha, Sci. Rep. 2, 759 (2012).

[8] M. Hase, I. Terasaki, and K. Uchinokura, Phys. Rev. Lett. 70, 3651 (1993). 
[9] M. Matsuda, K. Kakurai, J. E. Lorenzo, L. P. Regnault, A. Hiess, and G. Shirane, Phys. Rev. B 68, 060406 (2003).

[10] Y. Mizuno, T. Tohyama, S. Maekawa, T. Osafune, N. Motoyama, H. Eisaki, and S. Uchida, Phys. Rev. B 57, 5326 (1998).

[11] L. P. Regnault, M. Aïn, B. Hennion, G. Dhalenne, and A. Revcolevschi, Phys. Rev. B 53, 5579 (1996).

[12] R. O. Kuzian, S. Nishimoto, S. L. Drechsler, J. Málek, S. Johnston, J. van den Brink, M. Schmitt, H. Rosner, M. Matsuda, K. Oka, H. Yamaguchi, and T. Ito, Phys. Rev. Lett. 109, 117207 (2012).

[13] W. E. A. Lorenz, R. O. Kuzian, S. L. Drechsler, W. D. Stein, N. Wizent, G. Behr, J. Málek, U. Nitzsche, H. Rosner, A. Hiess, W. Schmidt, R. Klingeler, M. Loewenhaupt, and B. Büchner, Europhys. Lett. 88, 37002 (2009).

[14] G. Deng, V. Pomjakushin, V. Petříček, E. Pomjakushina, M. Kenzelmann, and K. Conder, Phys. Rev. B 84, 144111 (2011).

[15] A. Hisada, K. Matsubayashi, Y. Uwatoko, N. Fujiwara, G. Deng, E. Pomjakushina, K. Conder, D. Mohan Radheep, R. Thiyagarajan, S. Esakkimuthu, and S. Arumugam, J. Phys. Soc. Jpn. 83, 073703 (2014).

[16] G. Deng, N. Tsyrulin, P. Bourges, D. Lamago, H. Ronnow, M. Kenzelmann, S. Danilkin, E. Pomjakushina, and K. Conder, Phys. Rev. B 88, 014504 (2013).

[17] G. Deng, M. Kenzelmann, S. Danilkin, A. J. Studer, V. Pomjakushin, P. Imperia, E. Pomjakushina, and K. Conder, Phys. Rev. B 88, 174424 (2013).

[18] M. Matsuda, T. Yosihama, K. Kakurai, and G. Shirane, Phys. Rev. B 59, 1060 (1999).

[19] M. Matsuda and K. Katsumata, Phys. Rev. B 53, 12201 (1996).

[20] P. Abbamonte, G. Blumberg, A. Rusydi, A. Gozar, P. G. Evans, T. Siegrist, L. Venema, H. Eisaki, E. D. Isaacs, and G. A. Sawatzky, Nature (London) 431, 1078 (2004).

[21] S. Vanishri, D. Braithwaite, B. Salce, C. Marin, and J. Flouquet, Phys. Rev. B 81, 094511 (2010).

[22] R. S. Eccleston, M. Uehara, J. Akimitsu, H. Eisaki, N. Motoyama, and S. I. Uchida, Phys. Rev. Lett. 81, 1702 (1998).

[23] N. Motoyama, H. Eisaki, S. Uchida, N. Takeshita, N. Môri, T. Nakanishi, and H. Takahashi, Europhys. Lett. 58, 758 (2002).

[24] D. M. Radheep, R. Thiyagarjan, S. Esakkimuthu, G. Deng, E. Pomjakushina, C. L. Prajapat, G. Ravikumar, K.
Conder, G. Baskaran, and S. Arumugam, arXiv:1303.0921v1 (2013).

[25] G. Deng, N. Tsyrulin, M. Boehm, A. Ivanov, C. Rüegg, H. Ronnow, M. Kenzelmann, E. Pomjakushina, K. Conder, and G. J. McIntyre (unpublished).

[26] F. C. Zhang and T. M. Rice, Phys. Rev. B 37, 3759 (1988).

[27] G. Deng, D. M. Radheep, R. Thiyagarajan, E. Pomjakushina, S. Wang, N. Nikseresht, S. Arumugam, and K. Conder, J. Cryst. Growth 327, 182 (2011).

[28] D. Yu, R. A. Mole, and G. J. Kearley, EPJ Web Conf. 83, 03019 (2015).

[29] C. Ulrich, G. Deng, M. Yethiraj, S. J. Callori, S. Bruck, J. Bertinshaw, A. Brule, F. Klose, and G. J. McIntyre (unpublished).

[30] R. A. Ewings, A. Buts, M. D. Lee, J. v. Duijn, I. Bustinduy, and T. G. Perring, Nucl. Instrum. Methods Phys. Res., Sect. B 834, 132 (2016).

[31] C.-M. Wu, G. Deng, J. S. Gardner, P. Vorderwisch, W.-H. Li, S. Yano, J.-C. Peng, and E. Imamovic, J. Instrum. 11, P10009 (2016).

[32] J. E. Lorenzo, L. P. Regnault, C. Boullier, N. Martin, A. H. Moudden, S. Vanishri, C. Marin, and A. Revcolevschi, Phys. Rev. Lett. 105, 097202 (2010).

[33] R. Coldea, S. M. Hayden, G. Aeppli, T. G. Perring, C. D. Frost, T. E. Mason, S. W. Cheong, and Z. Fisk, Phys. Rev. Lett. 86, 5377 (2001).

[34] M. Nishi, O. Fujita, and J. Akimitsu, Phys. Rev. B 50, 6508 (1994).

[35] T. Huberman, R. Coldea, R. A. Cowley, D. A. Tennant, R. L. Leheny, R. J. Christianson, and C. D. Frost, Phys. Rev. B 72, 014413 (2005).

[36] M. Matsuda, H. Yamaguchi, T. Ito, C. H. Lee, K. Oka, Y. Mizuno, T. Tohyama, S. Maekawa, and K. Kakurai, Phys. Rev. B 63, 180403 (2001).

[37] M. Boehm, S. Coad, B. Roessli, A. Zheludev, M. Zolliker, P. Böni, D. M. Paul, H. Eisaki, N. Motoyama, and S. Uchida, Europhys. Lett. 43, 77 (1998).

[38] I. d. Pedro, J. M. Rojo, J. Rodriguez Fernández, M. T. Fernández-Díaz, and T. Rojo, Phys. Rev. B 81, 134431 (2010).

[39] N. Motoyama, T. Osafune, T. Kakeshita, H. Eisaki, and S. Uchida, Phys. Rev. B 55, R3386 (1997). 